\title{
ÉVALUATION DE L'EFFICACITÉ D'UNE MÉTHODE D'ÉCHANTILLONNAGE PAR NASSES DES ANGUILLES JAUNES (ANGUILLA ANGUILLA L.) EN MIGRATION DANS LA MEUSE.
}

\author{
E. BARAS, B. SALMON, J.C. PHILIPPART* \\ Laboratoire de Démographie des Poissons et d'Aquaculture, \\ Service d'Ethologie et de Psychologie Animales, \\ Université de Liège, 10 Chemin de la Justice, B 4500 TIHANGE, Belgique.
}

\begin{abstract}
RÉSUMÉ
Une méthodologie d'échantillonnage des anguilles jaunes en migration, basée sur l'utilisation de pièges mobiles (nasses "tonneau"; $100 \times 40 \mathrm{~cm} \varnothing$ ) immergés dans les bassins de repos de passes à poissons de type DENIL, a été testée en Meuse belge en 1993. Un maximum de 248 anguilles (taille moyenne : $292 \mathrm{~mm}$ ) a été recensé par unité d'effort de capture (nasse. bassin ${ }^{-1} \mathrm{j}^{-1}$ ). Dans une configuration standardisée, l'effectif de l'échantillon capturé dans les nasses est hautement prédictif $(R=0,998 ; 24$ ddl) de l'effectif total transitant par la passe à poissons. Les pièges s'avèrent non sélectifs à l'égard de la taille des anguilles jaunes $(114-613 \mathrm{~mm})$. L'application de cette méthodologie "d'échantillonnage ponctuel de migration" devrait permettre une caractérisation fiable de la dynamique de migration des anguilles dans des fleuves canalisés comme la Meuse.
\end{abstract} Meuse.

Mots-clés : Anguilla anguilla L., échantillonnage, migration, nasse, passe à poissons,

\section{EVALUATION OF A EEL-TRAP SAMPLING METHOD FOR THE ASSESSMENT OF MIGRANT YELLOW EELS ANGUILLA ANGUILLA (L.) IN THE RIVER MEUSE.}

\begin{abstract}
A sampling methodology using modified eel traps $(100 \times 40 \mathrm{~cm} \varnothing)$ installed in fish passes was evaluated for the assessment of migratory yellow eel Anguilla anguilla stock and population structure in the Belgian River Meuse in 1993. Up to 248 eels (mean size : $292 \mathrm{~mm}$ ) were captured in a single trap immersed overnight. In a standardized context, the size of the sample captured in the trap was highly predictive $(R=0.998 ; 24 \mathrm{df})$ of the number of eels migrating through the fish pass. The eel traps were evaluated as non selective towards fish size within the size range of yellow eels $(114-614 \mathrm{~mm})$. The delay for checking the eel traps $(2 \mathrm{~min})$ fits the purpose of frequent and numerous samples: Applying this "point migration sampling" methodology on a large scale would allow a reliable assessment of yellow eel migration dynamics in regulated rivers like the River Meuse.
\end{abstract}

Key-words : Anguilla anguilla L., sampling methodology, migration, eel-trap, fish pass, River Meuse.

\section{INTRODUCTION}

Les recherches menées au cours des 30 dernières années ont permis d'améliorer considérablement la compréhension du cycle de vie de l'anguille européenne, Anguilla anguilla (L.) (SINHA et JONES; 1975 ; DEELDER, 1984 ; BRUSLE, 1989a ; BERG, 1990). Paradoxalement; la phase dulçaquicole (stade anguille jaune); qui représente $\pm 90 \%$ de la durée du cycle de vie de l'espèce, est nettement moins connue que la migration des civelles (e.g. MORIARTY, 1990) ou que celle des anguilles argentées. Plusieurs auteurs

\footnotetext{
* Chercheur Qualifié du F.N.R.S.
} 
(TESCH, 1977 ; APRAHAMIAN, 1988 ; LEGAULT et PORCHER, 1990 ; NAISMITH et KNIGHTS, 1993) ont mis en évidence les variations des structures par taille, âge et sexe de l'aval à l'amont d'un système fluvial, mais à quelques exceptions près (MORIARTY, 1986 ; VØLLESTAD et JONSSON, 1988), la dynamique, la structure et l'intensité du flux migratoire des anguilles jaunes sont relativement méconnues. L'étude de ces aspects de la biologie de l'anguille s'avère pourtant essentielle pour la gestion des populations car la majorité des pêcheries d'anguilles ont enregistré une diminution des captures depuis 19791980 (BRUSLE, 1989b ; GUÉRAULT et DESAUNAY, 1990 ; MORIARTY, 1990 ; KNIGHTS et WHITE, 1994). Ce déclin des populations d'anguilles est particulièrement marqué dans les bassins des grands fleuves canalisés - où les barrages interfèrent avec la colonisation des eaux continentales par les anguilles jaunes (PHILIPPART et VRANKEN, 1983) - mais délicat à estimer en raison de la difficulté d'évaluer le renouvellement des stocks.

Dans le cadre d'un programme de recherches sur les stocks et les migrations des anguilles jaunes dans la Meuse belge et ses affluents (BARAS et al., in press), nous avons développé et évalué une méthodologie devant permettre de caractériser de manière fiable et rapide l'intensité du flux migratoire des anguilles et la structure par tailles de la population migrante, à l'échelle du cycle annuel ou du cycle journalier. Cette méthodologie se base sur l'utilisation de pièges mobiles, inspirés d'un modèle proposé par LEGAULT (1987) et installés dans les bassins de repos de passes à poissons équipant les barrages de navigation. Comme le signalent MORIARTY (1987) et LEGAULT (1987), les dispositifs de franchissement équipant les barrages permettent une discrimination fiable entre fractions migratrice et résidente de la population d'anguilles, du moins si on présuppose que l'utilisation de ces passes ne peut être systématiquement assimilée à l'expression de migrations trophiques journalières. Les tests présentés dans cette note concernent des échelles à ralentisseurs DENIL (1909), qui équipent plusieurs barrages sur le cours belge de la Meuse (PHILIPPART et al., 1988).

\section{MATÉRIEL ET MÉTHODE}

\section{Site d'études}

Notre étude a été réalisée sur le cours belge de la Meuse, au niveau du barrage d'Ampsin-Neuville (50 $32^{\prime} 00^{\prime \prime} \mathrm{N}, 5^{\circ} 17^{\prime} 26^{\prime \prime} \mathrm{E}, 32 \mathrm{~km}$ amont de Liège). Construit en 1958 et situé à $230 \mathrm{~km}$ de l'embouchure du fleuve, ce barrage représente le 11ème obstacle depuis l'embouchure du fleuve à la colonisation des eaux du bassin de la Meuse par des espèces amphibiotiques ( 7 obstacles aux Pays-Bas et 3 en Belgique à l'aval d'Ampsin-Neuville ; détails in PHILIPPART et al., 1994). A ce niveau, la Meuse est profonde de $5 \mathrm{~m}$ et son débit varie entre 16 et $2800 \mathrm{~m}^{3} \cdot \mathrm{s}^{-1}$ (1989-1993; source : Office de la Navigation). La présence de la centrale nucléaire de Tihange $(0,9 \mathrm{~km}$ en amont) est à l'origine d'un réchauffement sensible de l'eau $\left(2-5^{\circ} \mathrm{C}\right.$ selon les saisons). En 1993 , les extrêmes thermiques sont $4,5^{\circ} \mathrm{C}$ et $29,5^{\circ} \mathrm{C}$. La dénivellation du barrage est de $5 \mathrm{~m}$ au cours de l'étiage. Large de $140 \mathrm{~m}$, l'ouvrage est équipé de 5 vannes pertuis, d'une centrale hydroélectrique de $10000 \mathrm{~kW}$, de 2 écluses de navigation accollées et de 2 passes à poissons situées de part et d'autre du déversoir (détails in BARAS et al., 1994). Chaque passe (Fig. 1.A) comporte trois échelles (7,5 $\mathrm{m} \times 1,4 \mathrm{~m}$; pente : $24 \%$ ) constituées de ralentisseurs multiplans de type DENIL (1909), et séparées par deux bassins de repos $\left(7 \mathrm{~m}^{3}\right)$. Les débits dans les passes varient de 20 à $100 \mathrm{l} \cdot \mathrm{s}^{-1}$, correspondant à des vitesses de courant de 0,3 à $0,6 \mathrm{~m} \cdot \mathrm{s}^{-1}$.

\section{Méthodologie d'échantillonnage}

Les nasses utilisées (Fig. 1. B) sont inspirées du modèle de nasse «tonneau" (100 x $40 \mathrm{~cm} \varnothing$ ), décrit par LEGAULT (1987), construites en treillis métallique plastifié (maille $5 \mathrm{~mm}$ ) sur armature d'acier soudé à chaud ( 3 anneaux +4 lattes de renfort ; $4 \times 0,2 \mathrm{~cm}$ ). L'adjonction d'une porte coulissante (PVC, $20 \times 20 \mathrm{~cm}$ ) facilite la récolte des individus capturés. Les nasses sont immergées dans les 2 bassins de repos des échelles (1 nasse par bassin) et contrôlées le lendemain de leur immersion (contrôle entre 8h00 et $10 \mathrm{~h} 00$; GMT + 2). La durée moyenne de l'opération de récolte est de $2 \mathrm{mn}$ par nasse. Les contrôles ont été effectués quotidiennement du 28 mai au 1er juillet 1993, période correspondant à la migration des anguilles jaunes en Meuse' (BARAS et al., in press). 


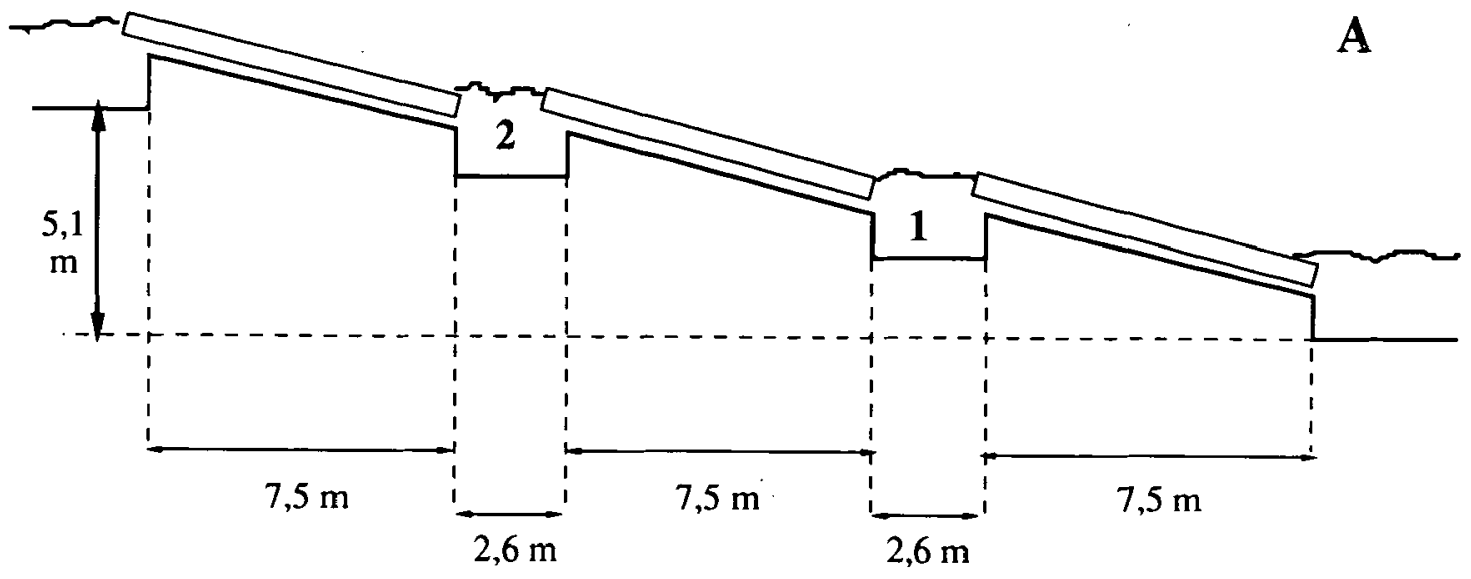

$\mathbf{B}$

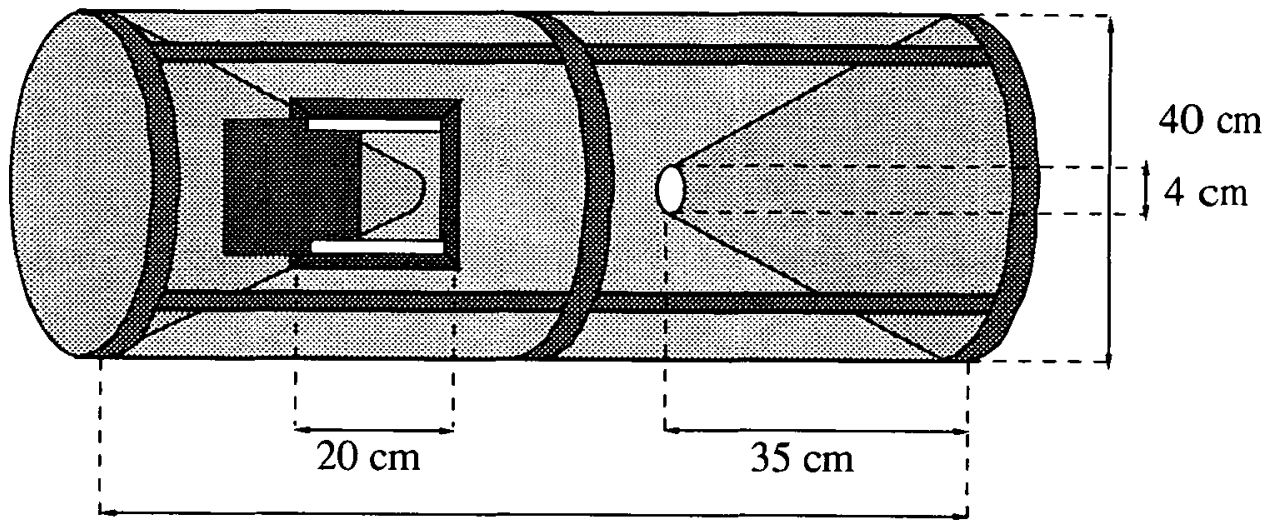

$100 \mathrm{~cm}$

Figure 1 : A. Section longitudinale de la passe à poissons à ralentisseurs DENIL du barrage d'Ampsin-Neuville (Meuse belge). 1 et 2 = bassins de repos où sont immergées les nasses. B. Treillis métallique plastifié (maille $5 \mathrm{~mm}$ ) sur armature d'acier soudé à chaud.

Figure 1 : A. Longitudinal section of a DENIL fish pass equipping the Ampsin-Neuville navigation weir on the Belgian River Meuse. 1 and $2=$ intermediate basins where are installed the eel traps. B. Coated wire mesh \# $5 \mathrm{~mm}$ on a welded steel brace.

Pour l'étude du rendement et de la sélectivité des nasses, les bassins de repos sont

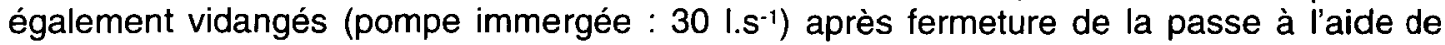
batardeaux. Le rendement de la nasse est défini comme le quotient du nombre d'anguilles capturées dans la nasse par le nombre total d'anguilles dans le bassin de repos (en nasse et hors nasse). En raison de l'absence de sélectivité de l'échelle DENIL vis-à-vis de la taille des anguilles (dans les conditions de débit précisées précédemment ; détails in BARAS et al., in press), les résultats des 2 bassins de repos ont été globalisés. Les anguilles, capturées à l'épuisette, sont anesthésiées (2-Phénoxy-éthanol : $0,4 \mathrm{ml}^{-1} \mathrm{I}^{-1}$ ), dénombrées et mesurées $( \pm 1 \mathrm{~mm})$. Pour chaque bassin, les longueurs moyennes des anguilles des 2 échantillons (nasse et bassin) sont comparées (t-tests de Student). L'effet de l'orientation des nasses a

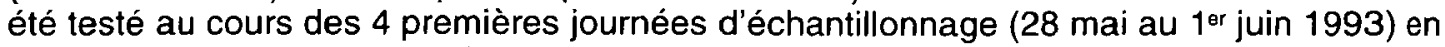
comparant les rendements de nasses immergées parallèlement et perpendiculairement à 
l'axe de l'échelle (1 nasse par bassin, orientations testées successivement). L'adéquation de la méthodologie à l'étude de la dynamique de migration des anguilles au cours du cycle journalier a été testée lors de 2 cycles de 24 heures au cours desquels trois nasses (1 par bassin de repos et 1 à l'entrée aval de l'échelle) étaient contrôlées toutes les 2 heures.

\section{RÉSULTATS}

\section{Orientation des nasses}

Les résultats des tests sur l'orientation des nasses sont présentés dans le Tableau I. Lorsque la nasse est orientée perpendiculairement à l'axe des ralentisseurs, le rendement des nasses varie entre 8,95 à $37,90 \%$ (moyenne $=27,2 \% ; N=4$ ), proportion significativement plus faible (Mann-Whitney $U$-test, $U=0, p<0,05$ ) que si la nasse est orientée parallèlement à l'axe du ralentisseur (rendement de 62,5 à $85,6 \%$; moyenne $=75,2 \% ; N=4$ ). Après ce test préliminaire, seule la dernière configuration a été retenue pour l'évaluation de la méthodologie.

\section{Sélectivité des nasses vis-à-vis de la taille des anguilles}

Du 28 mai au 1er juillet 1993, 6034 anguilles (114 - $645 \mathrm{~mm}$ ) furent capturées dans la passe à poissons en rive droite du barrage d'Ampsin-Neuville. La distribution des longueurs des anguilles capturées est pseudonormale, $97,9 \%$ des anguilles mesurant de 200 à $400 \mathrm{~mm}$ (moyenne : $298 \mathrm{~mm} ;$ S.D. $=43,6 \mathrm{~mm} ;$ Fig. 2). Les résultats des tests de sélectivité des nasses sont présentés dans le tableau I. Seuls 6 des 22 tests effectués révèlent que la longueur moyenne des anguilles capturées dans les nasses est significativement (seuil 0,05$)$ plus élevée. La différence de longueurs moyennes est toutefois faible (11 à $26 \mathrm{~mm}$ ). De plus, dans 3 autres cas, les anguilles capturées dans les nasses ont une longueur en moyenne inférieure à celle des anguilles hors nasses. Enfin, la distribution des longueurs de l'ensemble des anguilles capturées dans les nasses $(114-613 \mathrm{~mm}$ ) englobe celle des anguilles hors nasses (135 - $461 \mathrm{~mm})$. Ces différents éléments établissent l'absence de sélectivité des nasses vis-à-vis de la longueur des anguilles dans la gamme de longueurs considérées. Les mêmes tendances sont observées dans l'échelle en rive gauche du barrage (8 tests).

\section{Représentativité de l'effectif capturé dans les nasses}

Pour l'analyse de la représentativité de l'effectif capturé dans les nasses, nous avons exclu les journées où des modifications de débit, une orientation imparfaite de la nasse, une obstruction de la passe ou de l'entrée des nasses par des débris étaient susceptibles d'interférer avec le comportement des anguilles (soit un total de 25 couples d'échantillonnage pour l'échelle en rive droite du barrage). Le nombre maximum d'anguilles capturées par effort de capture (nasse. bassin ${ }^{-1}$. jour ${ }^{-1}$ ) est de 248 individus (taille moyenne : $292 \mathrm{~mm}$ ), pour un poids total de $9263 \mathrm{~g}$, correspondant à $\pm 29 \%$ du volume intérieur de la nasse. La relation entre l'effectif capturé dans les nasses et l'effectif total dans le bassin de repos est hautement significative $(F=3012 ; R=0,998 ; 24$ ddl, $p<0,001$; Fig. 3). Le caractère polynomial de la relation révèle une baisse de l'efficacité des nasses dans les situations de haute densité de population par bassin, reflétant probablement un début de saturation des nasses (capacité maximale théorique : \pm 850 anguilles de $300 \mathrm{~mm}$ ).

La précision du modèle a été testée pour l'échelle en rive gauche du barrage, où la même démarche expérimentale a été suivie du 9 juin au 16 juin 1993 (Tableau II). Le coefficient de corrélation $R$ de la relation entre valeurs prédites et observées est de 0,998 (15 ddl, $p<0,01)$ et la pente $(1,008)$ n'est pas significativement différente de 1 au seuil 0,05.

\section{Applicabilité à l'étude de la dynamique de migration au cours du cycle journalier}

Les distributions des effectifs capturés par nasse (1 nasse par bassin, 2 heures d'immersion) les 2-3 juin et 7-8 juin 1993 sont illustrés sur la figure 4 . Les nombres totaux d'anguilles capturées sont respectivement de 159 et 818 individus. Le rendement total des nasses dans ce protocole de relevés multiples varie entre 85,1 et $91,5 \%$, soit des valeurs nettement supérieures à celles observées au cours des tests précédents, étayant l'hypothèse de saturation des nasses dans des situations de forte densité de population. L'hypothèse d'échappement des anguilles au terme d'une phase d'attente ne peut toutefois être écartée, compte tenu du délai entre l'immersion des nasses et leur contrôle. Au cours 
Tableau I: Tests de sélectivité (t-tests de Student) vis-à-vis de la longueur des anguilles jaunes, des nasses installées dans les bassins des passes à poissons du barrage d'Ampsin-Neuville en 1993. (Bassins 1 et 2, cf. Fig. 1.A). (D) et (G) passes à poissons en rives droite et gauche. Journées où $\mathrm{N} 1$ ou $\mathrm{N} 2 \leq 5$ non illustrées. 28 mai - 1 ${ }^{\text {er }}$ juin : tests de l'effet de l'orientation des nasses. * : nasses orientées perpendiculairement à l'axe de l'échelle.

Table 1 : Tests of the selectivity towards fish size (Student t-tests) of the eel traps installed overnight in the pools of the Ampsin-Neuville fish passes in 1993 (pools 1 and 2 as in Fig. 1.A). (D) : right fish pass ; (G) : left fish pass. Days when N1 or N2 were $\leq 5$ are not presented. 28 May - 1 June : test of the orientation of the eel trap on catch-per-unit effort. * : traps oriented perpendicularly to the axis of the ladder.

Nombre et longueur corporelle $(\mathrm{mm})$ - Eel length $(\mathrm{mm})$ and number En nasses

(In eel traps)

Hors nasses

(Outside eel traps)

\begin{tabular}{|c|c|c|c|c|c|c|c|c|c|c|c|}
\hline Date & $\begin{array}{l}\text { Bassin } \\
\text { (Pool) }\end{array}$ & $\mathrm{N} 1$ & Moy. & SD & (Min-Max) & $\mathrm{N} 2$ & Moy. & SD & (Min-Max) & $t$ & $p$ \\
\hline 28 mai & $\begin{array}{l}1(D) \\
2(D)\end{array}$ & $\begin{array}{r}45 \\
125\end{array}$ & $\begin{array}{l}285 \\
306\end{array}$ & $\begin{array}{l}36,4 \\
37,0\end{array}$ & $(212-412)$ & $\begin{array}{l}27 \\
21\end{array}$ & $\begin{array}{l}282 \\
285\end{array}$ & $\begin{array}{l}32,9 \\
27,0\end{array}$ & & $\begin{array}{l}0,35 \\
2,43\end{array}$ & $\begin{array}{l}0,73 \\
0,02\end{array}$ \\
\hline 29 mai & $\begin{array}{l}{ }^{*} 1 \text { (D) } \\
{ }^{*} 2 \text { (D) }\end{array}$ & $\begin{array}{l}45 \\
96\end{array}$ & $\begin{array}{l}287 \\
305\end{array}$ & $\begin{array}{l}36,5 \\
41,5\end{array}$ & $\begin{array}{l}(206-371) \\
(233-425)\end{array}$ & $\begin{array}{l}131 \\
157\end{array}$ & $\begin{array}{l}288 \\
299\end{array}$ & $\begin{array}{l}37,5 \\
40,6\end{array}$ & $\begin{array}{l}(217-372) \\
(212-429)\end{array}$ & $\begin{array}{l}0,14 \\
1,25\end{array}$ & $\begin{array}{l}0,89 \\
0,21\end{array}$ \\
\hline 30 mai & $\begin{array}{l}{ }^{*} 1 \text { (D) } \\
{ }^{*} 2 \text { (D) }\end{array}$ & $\begin{array}{r}6 \\
20\end{array}$ & $\begin{array}{l}290 \\
315\end{array}$ & $\begin{array}{l}26,5 \\
43,1\end{array}$ & & $\begin{array}{l}61 \\
35\end{array}$ & $\begin{array}{l}301 \\
300\end{array}$ & $\begin{array}{l}42,1 \\
35,2\end{array}$ & & $\begin{array}{r}-0,61 \\
1,36\end{array}$ & $\begin{array}{l}0,54 \\
0,18\end{array}$ \\
\hline 01 juin & $\begin{array}{l}1(D) \\
2(D)\end{array}$ & $\begin{array}{l}249 \\
244\end{array}$ & $\begin{array}{l}302 \\
307\end{array}$ & $\begin{array}{l}32,8 \\
40,2\end{array}$ & $\begin{array}{l}(247-379) \\
(188-450)\end{array}$ & $\begin{array}{r}103 \\
53\end{array}$ & $\begin{array}{l}286 \\
305\end{array}$ & $\begin{array}{l}41,5 \\
46,5\end{array}$ & & $\begin{array}{l}2,57 \\
0,35\end{array}$ & $\begin{array}{l}0,01 \\
0,72\end{array}$ \\
\hline 02 & $\begin{array}{l}1 \text { (D) } \\
2 \text { (D) }\end{array}$ & $\begin{array}{r}204 \\
82\end{array}$ & $\begin{array}{l}303 \\
295\end{array}$ & $\begin{array}{l}48,3 \\
42,7\end{array}$ & & $\begin{array}{r}121 \\
41\end{array}$ & & & & $\begin{array}{l}1,77 \\
0,03\end{array}$ & $\begin{array}{l}0,08 \\
0,97\end{array}$ \\
\hline 09 juin & $\begin{array}{l}1(\mathrm{D}) \\
2(\mathrm{D}) \\
1(\mathrm{G}) \\
2(\mathrm{G})\end{array}$ & $\begin{array}{r}116 \\
63 \\
85 \\
42\end{array}$ & $\begin{array}{l}293 \\
304 \\
300 \\
294\end{array}$ & $\begin{array}{l}36,8 \\
38,0 \\
49,3 \\
32,8\end{array}$ & $\begin{array}{l}(204-3 \\
(217-3 \\
(200-4 \\
(232-3\end{array}$ & $\begin{array}{l}32 \\
29 \\
58\end{array}$ & $\begin{array}{l}289 \\
295 \\
283 \\
283\end{array}$ & $\begin{array}{l}37,2 \\
33,1 \\
46,7 \\
33,1\end{array}$ & 3) & $\begin{array}{l}0,54 \\
1,19 \\
1,63 \\
1,27\end{array}$ & $\begin{array}{l}0,59 \\
0,24 \\
0,10 \\
0,21\end{array}$ \\
\hline $10 j$ & $\begin{array}{l}1(\mathrm{D}) \\
2(\mathrm{D}) \\
1(\mathrm{G}) \\
2(\mathrm{G})\end{array}$ & $\begin{array}{r}115 \\
113 \\
13 \\
43\end{array}$ & $\begin{array}{l}295 \\
299 \\
319 \\
309\end{array}$ & $\begin{array}{l}38,5 \\
40,1 \\
70,9 \\
35,1\end{array}$ & $\begin{array}{l}(228-407) \\
(167-406) \\
(233-438) \\
(225-390)\end{array}$ & $\begin{array}{l}10 \\
18\end{array}$ & $\begin{array}{l}281 \\
285 \\
283 \\
295\end{array}$ & $\begin{array}{l}37,5 \\
35,0 \\
36,2 \\
56,7\end{array}$ & $\begin{array}{l}(172-352) \\
(201-345) \\
(230-340) \\
(233-430)\end{array}$ & $\begin{array}{l}2,29 \\
2,21 \\
1,56 \\
1,14\end{array}$ & $\begin{array}{l}0,02 \\
0,03 \\
0,13 \\
0,26\end{array}$ \\
\hline 11 juir & $\begin{array}{l}1 \text { (D) } \\
2 \text { (D) }\end{array}$ & $\begin{array}{r}148 \\
94\end{array}$ & $\begin{array}{l}296 \\
304\end{array}$ & $\begin{array}{l}44,5 \\
38,6\end{array}$ & & 60 & $\begin{array}{l}281 \\
298\end{array}$ & & & $\begin{array}{l}0,61 \\
0,76\end{array}$ & $\begin{array}{l}0,54 \\
0,45\end{array}$ \\
\hline 12 juin & $\begin{array}{l}1(\mathrm{D}) \\
2(\mathrm{D}) \\
1(\mathrm{G}) \\
2(\mathrm{G})\end{array}$ & $\begin{array}{l}216 \\
248 \\
188 \\
184\end{array}$ & $\begin{array}{l}278 \\
292 \\
299 \\
291\end{array}$ & $\begin{array}{l}45,8 \\
41,0 \\
46,0 \\
47,6\end{array}$ & $\begin{array}{l}(172-439) \\
(198-416) \\
(192-461) \\
(173-451)\end{array}$ & $\begin{array}{r}108 \\
178 \\
94 \\
99\end{array}$ & $\begin{array}{l}276 \\
281 \\
286 \\
274\end{array}$ & $\begin{array}{l}43,1 \\
42,0 \\
45,9 \\
41,8\end{array}$ & $\begin{array}{l}(152-436) \\
(135-404) \\
(171-425) \\
(171-392)\end{array}$ & $\begin{array}{l}0,38 \\
2,60 \\
2,29 \\
2,94\end{array}$ & $\begin{array}{l}0,71 \\
0,01 \\
0,02 \\
0,01\end{array}$ \\
\hline $13 \mathrm{ju}$ & $\begin{array}{l}1 \text { (D) } \\
2(\mathrm{D}) \\
1(\mathrm{G}) \\
2(\mathrm{G})\end{array}$ & $\begin{array}{r}231 \\
63 \\
47 \\
19\end{array}$ & $\begin{array}{l}279 \\
304 \\
324 \\
336\end{array}$ & $\begin{array}{l}40,4 \\
40,6 \\
57,5 \\
32,1\end{array}$ & $\begin{array}{l}(197-395) \\
(233-380) \\
(242-534) \\
(299-371)\end{array}$ & $\begin{array}{r}140 \\
29 \\
19 \\
6\end{array}$ & $\begin{array}{l}280 \\
295 \\
306 \\
307\end{array}$ & $\begin{array}{l}40,5 \\
39,8 \\
37,1 \\
34,7\end{array}$ & $\begin{array}{l}(170-396) \\
(211-418) \\
(227-382) \\
(250-404)\end{array}$ & $\begin{array}{r}-0,04 \\
0,87 \\
1,53 \\
1,79\end{array}$ & $\begin{array}{l}0,96 \\
0,39 \\
0,13 \\
0,09\end{array}$ \\
\hline 14 juin & $\begin{array}{l}1 \text { (D) } \\
2 \text { (D) }\end{array}$ & $\begin{array}{l}66 \\
47\end{array}$ & $\begin{array}{l}277 \\
308\end{array}$ & $\begin{array}{l}25,0 \\
47,6\end{array}$ & $\begin{array}{l}(235-328) \\
(207-428)\end{array}$ & $\begin{array}{l}21 \\
28\end{array}$ & $\begin{array}{l}279 \\
282\end{array}$ & $\begin{array}{l}27,3 \\
45,3\end{array}$ & $\begin{array}{l}(217-338) \\
(195-370)\end{array}$ & $\begin{array}{r}-0,36 \\
2,35\end{array}$ & $\begin{array}{l}0,72 \\
0,02\end{array}$ \\
\hline
\end{tabular}




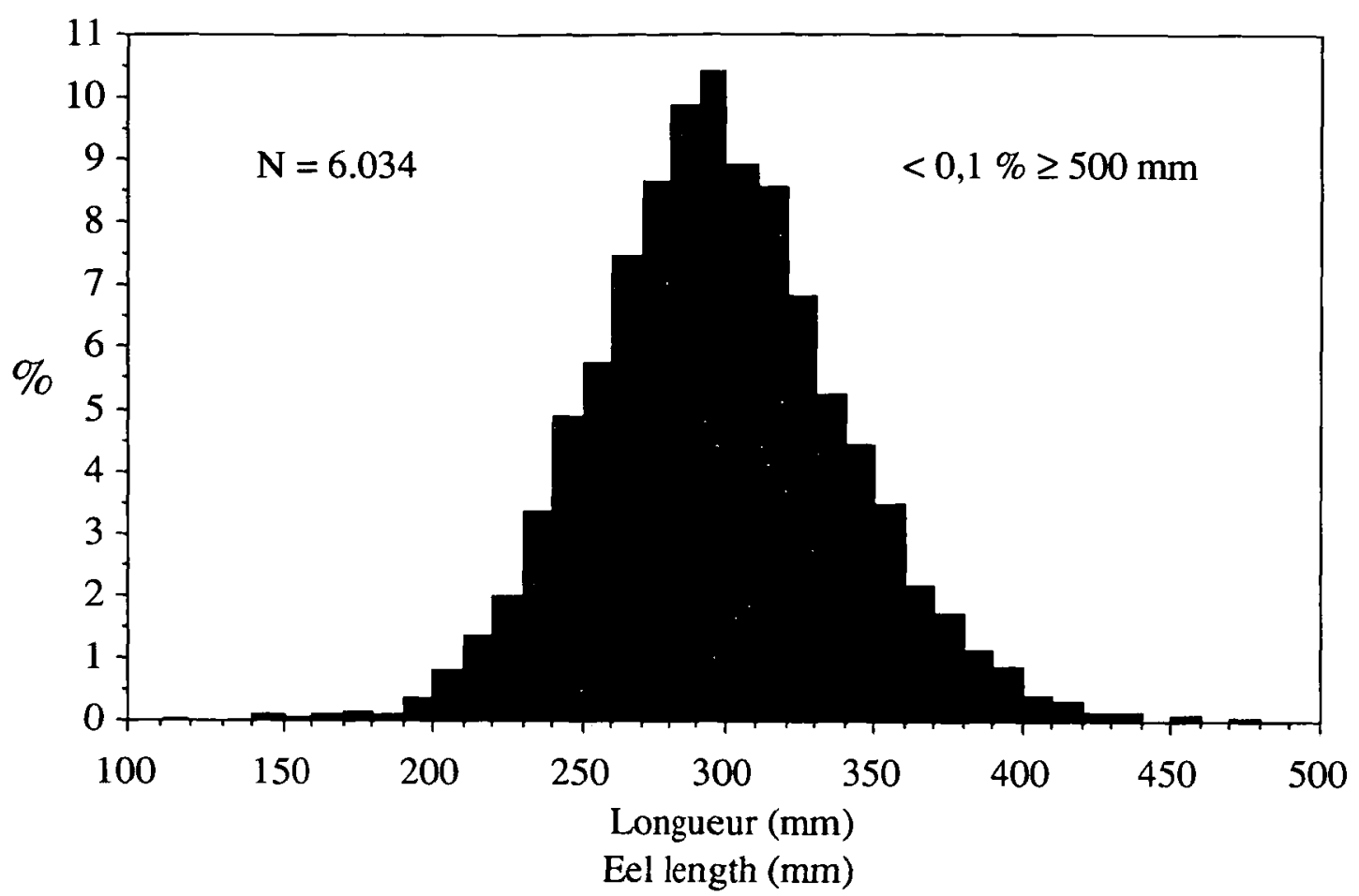

Figure 2 : Distribution des fréquences des longueurs des anguilles capturées dans la passe à poissons (type DENIL) en rive droite du barrage d'Ampsin-Neuville (28 mai - 1 juillet 1993).

Flgure 2 : Length frequency distribution of the yellow eels captured in the right DENIL fish pass of the Ampsin-Neuville navigation weir (28 May - 1 July 1993).

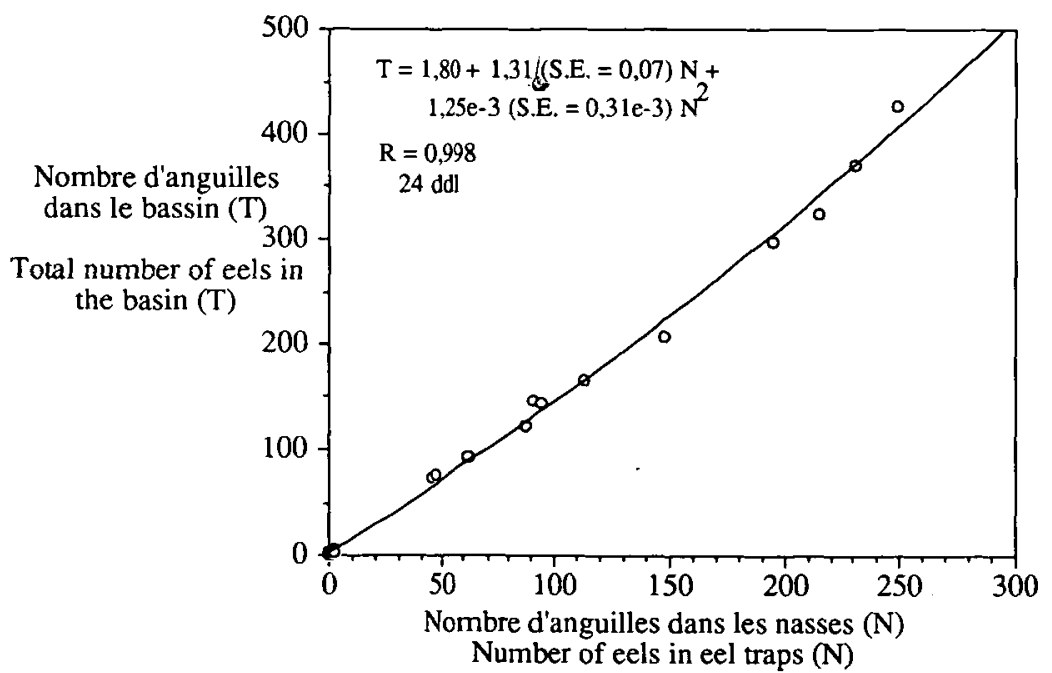

Figure 3 : Relation entre l'effectif d'anguilles capturé dans une nasse (immergée 24 h dans un bassin de repos d'une passe à ralentisseurs DENIL) et l'effectif total présent dans ce bassin (barrage d'Ampsin-Neuville, Meuse, 1993).

Figure 3 : Relationship between the number of eels captured in a trap immersed overnight in a basin of a DENIL fish pass and the total yield of eels in the basin (Ampsin-Neuville navigation weir, River Meuse, 1993). 
Tableau II : Test du modèle de représentativité de l'effectif capturé dans les nasses: (comparaison entre effectifs totaux prédits et observés dans les bassins de repos) dans la passe DENIL en rive gauche du barrage d'AmpsinNeuville (9 juin - 16 juin 1993).

Table II : Test of the model predicting total yield in a pool from the sample captured in eel traps (comparison between predicted and observed numbers) in the DENIL fish pass on the left side of the Ampsin-Neuville navigation weir (9 June - 16 June 1993).

\begin{tabular}{ccc}
\hline $\begin{array}{c}\text { Effectif en nasse } \\
\text { (Eel yield in eel trap) }\end{array}$ & $\begin{array}{c}\text { Effectif total en bassin (Total yield in pool) } \\
\text { Observé } \\
\text { (Observed) }\end{array}$ & $\begin{array}{c}\text { Prédit } \\
\text { (Predicted) }\end{array}$ \\
\hline 188 & 282 & 293 \\
184 & 283 & 286 \\
85 & 143 & 123 \\
47 & 66 & 66 \\
43 & 61 & 60 \\
42 & 64 & 59 \\
19 & 25 & 27 \\
13 & 23 & 19 \\
2 & 2 & 4 \\
2 & 3 & 4 \\
1 & 2 & 3 \\
0 & 4 & 2 \\
0 & 1 & 2 \\
0 & 1 & 2 \\
0 & 1 & 2 \\
0 & 0 & 954 \\
\hline
\end{tabular}

des 2 cycles effectués, la migration est quasi exclusivement nocturne : les nombres d'anguilles capturées avant le coucher et après le lever du soleil sont faibles (5 et 29 individus, soit 3,1 et $3,5 \%$ du total journalier). Dans les bassins de repos, le nombre d'anguilles capturées dans les nasses croît de manière continue au cours de la nuit et atteint une valeur maximale entre $4 \mathrm{hOO}$ et $6 \mathrm{hOO}$. Par contre, la distribution des captures dans les nasses à l'aval de l'échelle est bimodale (22h00-24h00 et 4h00-6h00), ce qui correspond aux observations de LEGAULT (1987). Cette différence est probablement imputable à des dynamiques de migration différentes en milieu ouvert (aval de l'échelle) et fermé (bassins de repos). La même hypothèse peut être retenue pour expliquer la variabilité des effectifs capturés dans les bassins de repos et en aval de l'échelle (maxima respectifs : 138 et 55 individus). Si nous considérons que l'ensemble des anguilles capturées dans la passe à poissons ont rencontré la nasse immergée dans le chenal d'entrée aval de la passe à poissons, le rendement de cette nasse en milieu ouvert est nettement plus faible que dans les bassins $(8,1-13,9 \%$ vs. $85,1-91,5 \%)$.

\section{DISCUSSION ET CONCLUSIONS}

D'après les résultats des tests effectués, la méthodologie d'échantillonnage par nasses dans les échelles à poissons se révèle parfaitement adaptée à l'étude de la migration des anguilles jaunes : les nasses s'avèrent peu ou pas sélectives vis-à-vis de la longueur des individus dans une gamme représentative de celle des anguilles jaunes (DEELDER, 1984 ; MORIARTY, 1987) et l'effectif capturé est hautement représentatif du nombre total d'anguilles dans le bassin de repos. De par sa rapidité d'exécution et son caractère non perturbateur, la méthodologie des nasses peut également s'avérer intéressante pour l'étude de la dynamique de migration des anguilles à l'échelle du cycle journalier. 

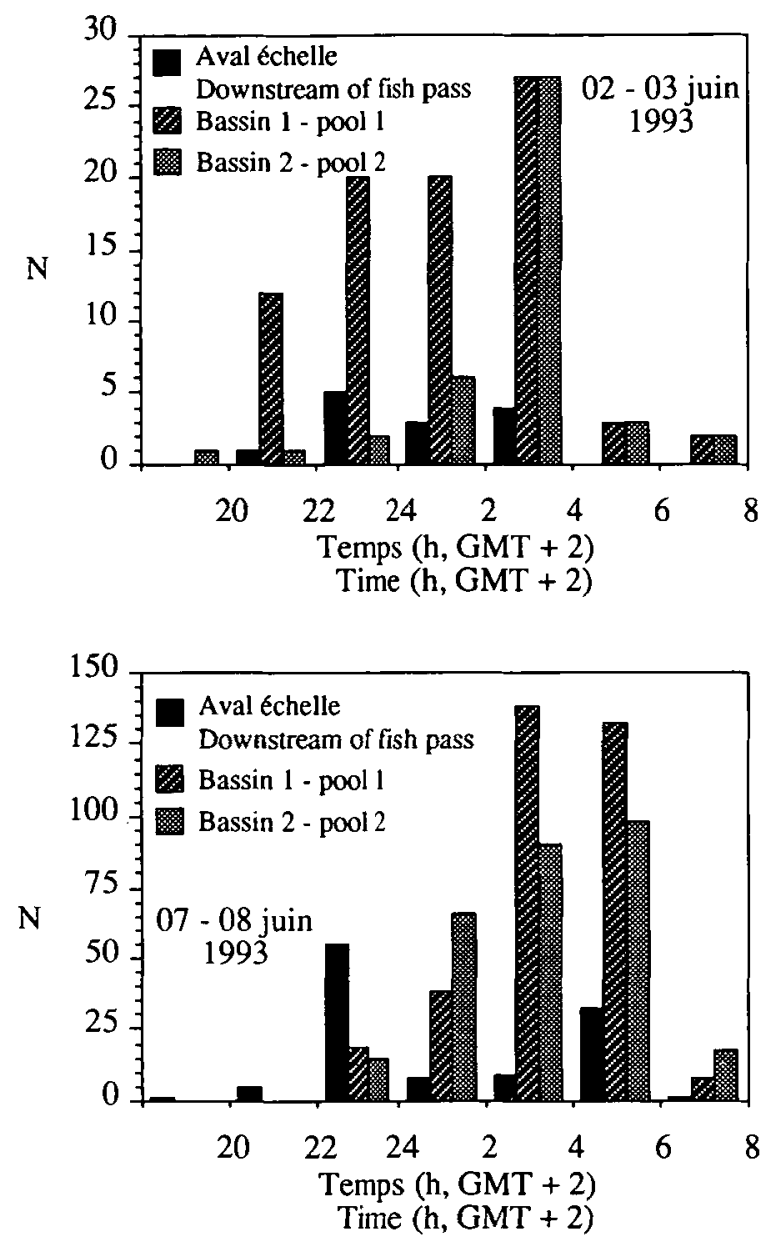

Figure 4 : Variations, au cours de 2 cycles journaliers, du nombre d'anguilles capturées dans les nasses installées dans la passe à poissons (type DENIL) en rive droite du barrage d'Ampsin-Neuville (bassins 1 et 2, cf. Fig. 1.A). Le contrôle de 20 h00 couvre la période 10 h00 - 20 h00.

Figure 4 : Variations of the number of eels captured in three eel traps installed in the right DENIL fish pass of the Ampsin-Neuville navigation weir (basins 1 and 2 as in Fig. 1.A). The 20 h00 control covers a $10 \mathrm{~h}$ period (10h00-20h00).

Ainsi que l'ont mis en évidence les tests sur l'orientation des nasses et l'analyse de leur rendement en milieu ouvert, un positionnement standardisé des nasses s'avère strictement nécessaire à toute inférence sur l'intensité du flux migratoire. La portée du modèle présenté est donc strictement limitée à des bassins de repos de passes DENIL comparables à celles où les tests ont été effectués. La transposition de cette méthodologie à d'autres types de passes nécessiterait une calibration préalable du rendement des nasses en fonction de l'environnement, mais il est permis de supposer que la fiabilité de cette calibration serait suffisante pour la détermination de l'intensité du flux migratoire d'anguilles jaunes dans la passe. Appliquée à l'échelle d'un fleuve canalisé comme la Meuse, cette méthodologie "d'échantillonnage ponctuel de migration» constituerait un outil fiable pour l'étude de la dynamique de migration des anguilles, de même que pour l'évaluation de l'incidence des obstacles physiques et de l'efficacité des dispositifs de franchissement installés, lesquels 
peuvent s'avérer extrêmement variables (LEGAULT, 1992 ; PORCHER, 1992). Dans ce contexte, et de manière à effectuer une discrimination précise entre la migration anadrome et des mouvements trophiques saisonniers ou journaliers dans un domaine vital incluant la zone d'influence du barrage, il importerait d'apporter des précisions complémentaires sur ces mouvements journaliers, notamment via un programme de marquage des anguilles capturées (e.g. injection de colorant par Panjet; NIELSEN, 1988) couplé à un suivi par biotélémétrie (e.g. LABAR et al., 1987).

\section{REMERCIEMENTS}

Cette étude a été réalisée dans le cadre d'un programme «Anguille» financé par la Commission Provinciale de Liège du Fonds Piscicole en 1992-1993. Pour leur participation au travail sur le terrain, nous remercions G. RIMBAUD et J.M. LAMBERT.

\section{BIBLIOGRAPHIE}

APRAHAMIAN M.W., 1988. Age structure of eel, Anguilla anguilla (L.), populations in the River Severn, England, and the River Dee, Wales. Aquacult. Fish. Manage., 19, 365-376.

BARAS E., LAMBERT H., PHILIPPART J.C., 1994. A comprehensive assessment of the failure of Barbus barbus (L.) migrations through a fish pass in the canalized River Meuse (Belgium). Aquat. Liv. Resour., 7 (3), 181-189.

BARAS E., PHILIPPART J.C., SALMON B., in press. Estimation of migrant yellow eel stock in large rivers through the survey of fish passes. - A preliminary approach in the River Meuse (Belgium). Fish. Manage. Ecol., in press.

BERG R., 1990. The growth of eels : a critical assessment of data from open waters. Presented at EIFAC Working Party on Eel, Porto (Portugal), 31 May-3 June 1989. Int. Rev. Ges.. Hydrobiol., 75 (6), 755-762.

BRUSLE J., 1989a. Les migrations du cycle biologique des anguilles : de l'aire de ponte marine à la colonisation des milieux continentaux (leptocéphales, civelles, anguilles jaunes). Oceanis, 15 (2), 181-195.

BRUSLE J., 1989b. L'anguille européenne (Anguilla anguilla), une espèce jugée commune jusqu'à la dernière décennie, mérite-t-elle d'acquérir aujourd'hui le statut d'espèce menacée ? Bull. Soc. Zool. France, 114 (3), 61-70.

DEELDER C.L., 1984. Synopsis of biological data on the eel Anguilla anguilla (Linnaeus, 1758). FAO Fisheries Synopsis, $n^{\circ} 80(\operatorname{rev} 1), 73 p$.

DENIL G., 1909. Les échelles à poissons et leurs applications aux barrages de Meuse et l'Ourthe. Bull. Acad. Sci. Belg., 1221-1224.

GUÉRAULT D, DESAUNAY Y., 1990. The evolution of recruitment of glass eel (Anguilla anguilla) in the Loire estuary, France, from 1977 to 1988. Presented at EIFAC Working Party on Eel, Porto (Portugal), 31 May-3 June 1989. Int. rev. Gesamt. Hydrobiol., 75 (6), 717-719.

KNIGHTS B., WHITE E., 1994. Stock assessment of European eel Anguilla anguilla L. In COWX I.G., Abstracts of the Communications presented in the International Symposium and Workshop on Stock Assessment in Inland Fisheries, University of Hull, 11-15 April 1994, 27, HIFI Press, Hull, England.

LABAR G.W., HERNANDO CASAL J.A., DELGADO C.F., 1987. Local movements and population size of European eels, Anguilla anguilla, in a small lake in Southwestern Spain. Env. Biol. Fishes, 19 (2), 111-117.

LEGAULT A., 1987. L'anguille dans le bassin de la Sèvre Niortaise. Publ. Dép. Halieut. Ecol. Nat. Sup. Agron. Halieut., 6, $295 \mathrm{p}+$ ann. 
LEGAULT A., 1992. Etude de quelques facteurs de sélectivité des passes à anguilles. Bull.Fr. Pêche Piscic., 325, 83-91.

LEGAULT A., PORCHER J.P., 1990. Distribution of eel stocks in Brittany. Presented at EIFAC Working Party on Eel, Porto (Portugal), 31 May-3 June 1989. Int. Rev. Ges.. Hydrobiol., 75 (6), 795.

MORIARTY C., 1986. Riverine migration of young eels Anguilla anguilla (L.). Fish. Res., 4(1), 43-58.

MORIARTY C., 1987. The European eel - Discoveries and developments. In CROZIER W.W. et JOHNSTON P.W. (eds) Proceedings of the 17th Ann. Study Course Inst. Fish. Manage. (Univ. Ulster, Coleraine, 9-11 September 1986), 100-110.

MORIARTY C., 1990. European catches of elver of 1928-1988. Presented at EIFAC Working Party on Eel, Porto (Portugal), 31 May-3 June 1989. Int. Rev. Ges.. Hydrobiol., 75 (6), 701-706.

NAISMITH I.A., KNIGHTS B., 1993. The distribution and growth of the eel, Anguilla anguilla, in the freshwater catchment of the River Thames. J. Fish Biol., 42 (2), 217-226.

NIELSEN J., 1988. A review of marking and tagging methods applied to eel (Anguilla anguilla L.). EIFAC Occasional Paper, 21, $24 \mathrm{p}$.

PHILIPPART J.C, VRANKEN, M., 1983. I'Anguille. p. 55-61 in Atlas des poissons de Wallonie. Distribution, écologie, éthologie, pêche, conservation. Cah. Ethol. appl., 3(suppl. 1-2), $395 \mathrm{p}$.

PHILIPPART J.C., GILLET A., MICHA J.C., 1988. Fish and their environment in large European river ecosystems : the River Meuse. Sciences de l'eau, 7(1), 115-154.

PHILIPPART J.C., MICHA J.C., BARAS E., PRIGNON C., GILLET A., JORIS S., 1994. The Belgian Project "Meuse Salmon 2,000" - First results, problems and future prospects. Water Science and Technology, 29 (3), 315-317.

PORCHER J.P., 1992. Les passes à anguilles. Bull. Fr. Pêche Piscic., 326-327, 134-142.

SINHA V.R.P., JONES J.W., 1975. The European Freshwater Eel. Liverpool University Press, Liverpool, $146 \mathrm{p}$.

TESCH F.-W., 1977. The Eel. Biology and Management of Anguillid Eels. Chapman and Hall, London, $434 \mathrm{p}$.

VØLLESTAD L.A., JONSSON B., 1988. A 13 year study of the population dynamic and growth of the European eel Anguilla anguilla in a Norwegian river : evidence for density-dependent mortality, and development of a model for predicting yield. J. Anim. Ecol., 57, 983-997. 